\title{
Containment measures limit environmental effects on COVID-19 early outbreak dynamics
}

Gentile Francesco Ficetola ${ }^{1,2, *}$ and Diego Rubolini ${ }^{1 *}$

${ }^{1}$ Dipartimento di Scienze e Politiche Ambientali, Università degli Studi di Milano, via Celoria 26, I-20133 Milano, Italy

${ }^{2}$ Université Grenoble Alpes, CNRS, Université Savoie Mont Blanc, LECA, Laboratoire

d'Ecologie Alpine, F-38000 Grenoble, France

*Contributed equally to this work; order was decided with a coin toss

Correspondence: francesco.ficetola@gmail.com,diego.rubolini@unimi.it 
Abstract: Environmental factors are well known to affect spatio-temporal patterns of infectious disease outbreaks, but whether the recent rapid spread of COVID-19 across the globe is related to local environmental conditions is highly debated. We assessed the impact of environmental factors (temperature, humidity and air pollution) on the global patterns of COVID-19 early outbreak dynamics during January-May 2020, controlling for several key socio-economic factors and airport connections. We showed that during the earliest phase of the global outbreak (January-March), COVID-19 growth rates were non-linearly related to climate, with fastest spread in regions with a mean temperature of ca. $5^{\circ} \mathrm{C}$, and in the most polluted regions. However, environmental effects faded almost completely when considering later outbreaks, in keeping with the progressive enforcement of containment actions. Accordingly, COVID-19 growth rates consistently decreased with stringent containment actions during both early and late the early variation among regions in disease spread. With limited policy interventions, seasonal patterns of disease spread might emerge, with temperate regions of both hemispheres being most at risk of severe outbreaks during colder months. Nevertheless, containment measures play a much stronger role and overwhelm impacts of environmental variation, highlighting the key role for policy interventions in curbing COVID-19 diffusion within a given region. If the disease will become seasonal in the next years, information on environmental drivers of COVID-19 can be integrated with epidemiological models to inform forecasting of future outbreak risks and improve management plans.

\section{Keywords:}

Temperature; absolute humidity; COVID-19, pathogen growth rate; global analysis; Climate; Population size; Pollution; PM 2.5 


\section{Introduction}

Host-pathogen interaction dynamics can be significantly affected by environmental conditions, either directly, via e.g. improved pathogen transmission rates, or indirectly, by affecting host susceptibility to pathogen attacks (Altizer et al., 2013). In the case of directly transmitted diseases, such as human influenza and other viral diseases, multiple environmental parameters including local temperatures and humidity impact on virus viability and transmission, with significant consequences for the seasonal and geographic patterns of outbreaks (Shaman and Kohn, 2009; Fuhrmann, 2010; Shaman et al., 2010; Lowen and Steel, 2014; Kampf et al., 2020). The coronavirus SARS-CoV-2 is the aethiological agent of COVID-19, a pandemic zoonosis causing severe pneumonia outbreaks at a global scale (World Health Organization, 2020). During the initial months of 2020, this disease rapidly spread worldwide (Dong et al., 2020), though the early dynamics of COVID-19 outbreaks appeared highly variable. Some countries were experiencing slow growth and spread of COVID-19 cases, while others were suffering widespread community transmission and fast, nearly exponential growth of infections (Dong et al., 2020). Understanding the environmental drivers of early growth rates is pivotal to forecast the potential severity of disease outbreaks and their interactions with containment measures (Britton and Tomba, 2019; Baker et al., 2020; Jung et al., 2020). Given the importance of environmental conditions on the transmission of many pathogens, we tested the hypothesis that the severity of COVID-19 outbreaks across the globe was affected by spatial variation of key environmental factors, and investigated the relative role of environmental conditions and of containment measures adopted by governments on disease spread patterns. 

growth rate and multiple environmental features, such as temperature, humidity (e.g. Tamerius et al., 2013; Islam et al., 2020a; Kampf et al., 2020; Runkle et al., 2020; Sajadi et al., 2020; Sobral et al., 2020; Wu et al., 2020c), and air pollution (e.g. Bianconi et al., 2020; Rahman et al., 2020;

\section{.} (5) possibility of correctly identifying environmental signals on COVID-19 spread dynamics (Carlson et al., 2020a; Carlson et al., 2020b). Differences among studies can be caused by multiple factors, including lack of standardized methodological framework, differences in spatial extent and scale, and by complex interactions between human transmission, environmental features and containment measures (Baker et al., 2020; Carlson et al., 2020b). Furthermore, both environmental features and containment measures can show complex temporal trends in the course of an outbreak. Studies assessing whether relationships between environment and COVID-19 change are consistent across regions and time periods are pivotal to identify robust and generalizable patterns.

We calculated the mean daily growth rate of confirmed COVID-19 cases during the exponential phase of the epidemic growth curve for the 586 countries/regions (hereafter, regions) (Supplement 1, Fig. S1) where at least 25 cases were reported before June, 2020. Variation at these early epidemic growth rates represents the local progression of the disease and should best reflect the impact of local environmental conditions on disease spread. However, environmental effects on local disease spread could be blurred by containment actions, as in most regions local authorities adopted unprecedented containment measures well in advance or immediately after 

et al., 2020; Maier and Brockmann, 2020; Manenti et al., 2020; Thu et al., 2020).

In this study, we first assessed whether COVID-19 growth rate in different regions of the world was affected by major environmental features (temperature, humidity, fine particulate matter; see Methods), controlling for major socio-economic features of the affected regions. rate at the onset of local outbreaks (Maier and Brockmann, 2020). Among the socio-economic factors potentially affecting SARS-CoV-2 transmission dynamics during early outbreaks, we (hereafter, health expenditure) and age structure (see Methods). The importance of a given region in the global air transportation network was expressed as its eigenvector centrality (Coelho et al., 2020) (hereafter, region centrality; see Methods) while containment measures 


\subsection{COVID-19 dataset}

We downloaded time series of confirmed COVID-19 cases (cumulative growth curves) from the Johns Hopkins University Center For Systems Science and Engineering (JHU-CSSE) GitHub repository (https://github.com/CSSEGISandData/COVID-19/) (Dong et al., 2020). JHU-CSSE reports, for each day since January 22, 2020, confirmed COVID-19 cases at the country level or at the level of significant geographical units belonging to the same country, which we broadly defined here as 'regions' (e.g. US states, or China and Canada provinces; Supplement 1, Supplementary methods). Data referring to outbreaks occurring on cruise ships were not considered. The cumulative growth curves were carefully checked and obvious reporting errors (a few occurrences of temporary decreases in the cumulative number of cases) were corrected. Our dataset included confirmed COVID-19 cases up to June 15, 2020. From this dataset, we selected data for all those regions in which local outbreaks were detected up to May 31, 2020 (see Local outbreaks and COVID-19 cases growth rates).

Overall, we considered data from 159 countries. We considered sub-national level data for the all the countries of the world for which data were easily accessible from the original sources listed in the JHU-CSSE website (for a total of 17 countries; Table S6). Our final dataset included information on 586 regions (Supplement 1, Fig. S2 and Supplementary methods).

\subsection{Local outbreaks and COVID-19 cases growth rates}

To avoid the biases arising because of incomplete spread of the pathogen, our dataset included only those regions experiencing a local COVID-19 outbreak. Therefore, our results are unaffected by patterns occurring in regions where the pathogen showed a limited number of 
records (e.g. because of distributional disequilibrium, limited connections with other affected areas, or lack of reporting).

The onset of a local COVID-19 outbreak event was defined as the day when at least 25 confirmed cases were reported in a given region. Visual inspection of growth curves showed that, in most cases, below this threshold the reporting of cases was irregular, or growth was extremely slow for prolonged periods. This approach also allowed us to exclude the first cases, often referring to individuals returning from foreign countries and not reflecting local transmission of the pathogen. We then calculated the daily growth rate $r$ of confirmed COVIDproposed by Hall et al. (2014). The method iteratively fits growth curves on successive intervals of a minimum of 5 data points to identify the exponential phase of a cumulative growth curve, and returns the lag phase, and the onset and end of the exponential growth phase. The lag phase, characterized by very slow growth, is followed by the exponential phase (Supplement 1, Fig. S1). Typically, cumulative growth curves of COVID-19 cases begin with exponential growth in the early phases, which begins to decelerate within ca. 10 days of its beginning (e.g. Supplement 1, Fig. S3; see also Maier and Brockmann, 2020). This pattern is similar to what has been documented for earlier phases of other major infectious disease outbreaks (Viboud et al., 2016). We thus restricted the analyses to those regions for which at least 15 days of data after the outbreak onset were available up to June 15, 2020.

Approaches assuming distributional equilibrium can be inappropriate to model the spread of recently emerged infectious diseases (Carlson et al., 2020a). To avoid this issue, we used a dynamic approach, whereby we modelled the dynamics of disease spread within populations (Hall et al., 2014; Carlson et al., 2020a; Coelho et al., 2020). To this end, we computed the mean 
daily growth rate of confirmed COVID-19 cases during the exponential phase as $r=[\ln (n$ cases $\left._{\text {day end exp. phase }}\right)-\ln \left(\right.$ n cases $\left.\left._{\text {day start exp. phase }}\right)\right] /($ day end exp. phase - day end exp. phase). We also computed the maximum daily growth rate $r_{\max }$ during the exponential phase according to Hall et al. (2014). Lag and exponential phase duration, and $r_{\max }$ were computed through the R package growthrates (Hall et al., 2014). Mean and maximum daily growth rates were strongly positively correlated (Pearson's correlation coefficient, $\mathrm{r}=0.95, \mathrm{n}=586$ regions), indicating that our growth rate estimates for a given region were highly consistent irrespective of the method used for calculations. By modelling the exponential phase, this approach allowed to focus on local transmission events occurring within the focal region. The average time interval between growth rate estimates.

\subsection{Environmental variables}

We considered two climatic variables that are known to affect the spread of viral diseases: mean air temperature and specific humidity (water vapor pressure), which is a measure of absolute humidity. Previous studies showed that, for coronaviruses and influenza viruses, survival is generally higher at low temperature and low values of absolute humidity (Lowen et al., 2007; et al., 2020). For each region, we obtained the mean daily values for temperature $\left({ }^{\circ} \mathrm{C}\right)$ and specific humidity $\left(\mathrm{g} / \mathrm{m}^{3}\right)$ from the ERA5 hourly database (Supplement 1, Supplementary methods). 
The latency period of the infection, and the lag time between the onset of symptoms,

PCR tests and publication of confirmed cases can be highly variable across patients and across areas of the world. For instance, Li et al. (2020a) suggested a mean incubation period of 4-7 days, but also reported cases with shorter incubation, or with incubation $>14$ days. Therefore, we measured the potential impact of temperature and humidity in two alternative time windows. First, we considered a broad time period (30 days) occurring before the end of exponential phase. For this 30-days time period, we computed mean climatic conditions (temperature and humidity during 30 days; including the day of the end of the exponential phase and the preceding 29 days; hereafter: 30-days period) (Supplement 1, Fig. S1). This 30-days period aims at covering all the climatic conditions encountered by the broadest range of confirmed cases. Second, we used a narrower time period, focusing on the most frequent time lags between infection and reporting. Following Jüni et al. (2020), we computed mean climatic values assuming an exposure period for infections starting 14 days before the onset of the follow-up period (in our case the start of the exponential phase) and ending 14 days before the end of the follow-up period (in our case the end of the exponential phase) (hereafter: $\Delta 14$ days period) (Supplement 1 , Fig. S1).

Besides climate, it has been proposed that other environmental parameters may affect variation of COVID-19 outbreak severity. Air pollution, especially fine atmospheric particulate, may enhance the environmental persistence, transmission and effects of coronaviruses (Bianconi et al., 2020; Zhang et al., 2020). We thus calculated the mean annual concentration of PM2.5 for each region (Supplement 1, Supplementary methods). 
Among socio-economic predictors, we considered mean human population density (Center for International Earth Science Information Network, 2018) (hereafter, population density, expressed in inhabitants $/ \mathrm{km}^{2}$ ), total population size (Center for International Earth Science Information Network, 2018), per capita government health expenditure (in US\$; average of 2015-2017 values) (Supplement 1, Supplementary methods). Elderly people are more susceptible to develop severe COVID-19 symptoms (Wu et al., 2020a). We thus obtained for each country an estimate of the proportion of the population aged 65 or older (population $65+$ ). (Pybus et al., 2015), and such an effect has been highlighted also for early SARS-Cov-2 spread global airport connections and COVID-19 growth rate. Highly connected regions may experience a higher 'propagule pressure' that increase disease diffusion among hosts, ultimately influencing disease growth rates (Coelho et al., 2020). To investigate whether airport connections affected early COVID-19 growth rates, we computed the eigenvector centrality score for each region (region centrality). Highly connected regions have a higher region centrality score (Bonacich, 1987) (Supplement 1, Supplementary methods).

\subsection{Stringency of containment measures}

For each region, we obtained an index of the overall stringency of COVID-19 containment measures adopted by local authorities in the corresponding country at the onset of a local outbreak (hereafter, stringency index). The stringency index was obtained by combining information for each country from two separate data sources (Supplement 1, Supplementary methods). This index simply record the number and strictness of government response measures, 
hence a higher stringency score does not necessarily imply that a country's response is more effective than that of other countries with lower scores (Hale et al., 2020). Nevertheless, the stringency index may be helpful to illustrate the timeline of interventions and to assess whether local governments' policy responses at outbreak onset had any impact on COVID-19 spread 210 within a given region.

\subsection{Statistical analyses}

We relied on linear mixed models (LMMs) to relate variation of COVID-19 growth rate across regions to environmental and socio-economic/management predictors (temperature, humidity,

PM2.5, population density, population size, health expenditure, population $65+$, region centrality, stringency index). LMMs are an extension of linear models that allow to take into account non-independence of data (Zuur et al., 2009). In our study case, multiple regions within a given country were considered as non-independent as they share multiple features (e.g. health policy, monitoring protocols, economic features other than those considered in the analyses). Country identity was thus included as a random factor to account for non-independence of growth rates from regions belonging to the same country. Non-linear relationships between climatic factors and ecological variables are frequent (Legendre and Legendre, 2012), and have also been suggested for relationships between SARS-CoV-2 occurrence and climate (e.g. Runkle et al., 2020). As in exploratory plots we detected a clear non-linear relationship between $r$-values and climate variables, we included in models both linear and quadratic terms. Humidity, PM2.5, population density, population size, health expenditure and region centrality were $\log _{10^{-}}$ transformed to reduce skewness and improve normality of residuals. Regression models can be heavily affected by strong collinearity among predictors $(|\mathrm{r}| \sim 0.70$ or above) (Dormann et al., 
2013). In our dataset, temperature and humidity showed a very strong positive correlation (Supplement 1, Fig. S7 and Table S1). We thus fitted separate models for temperature and

To assess temporal variation in the importance of different predictors on COVID-19

growth rates, we fitted LMMs considering regions experiencing outbreaks in different periods.

Each LMM included data from regions experiencing outbreaks up to a given day. We started cumulative curve in Supplement 1, Fig. S4). The partial $R^{2}$ statistic (variance explained by each fixed effect, or semi-partial $R^{2}$ ) was taken as a measure of the importance of each fixed effect in each of these models. Furthermore, we assessed temporal variation of standardized regression coefficients for models fitted at different time points. Airport connections are expected to affect the first phases of the epidemic events, and we therefore tested the effect of region centrality in a model including data up to March 15, 2020 (Supplement 1, Supplementary results). To confirm the time lag period used for the calculation of temperature and humidity (30-days period vs. $\Delta 14$ days period) did not affect our results, we repeated analyses twice, first using the 30-days period data, and then using the $\Delta 14$ days period data. Climate variables calculated using the 30 -days and the $\Delta 14$ days periods showed almost perfect correlation across regions (temperature, $\mathrm{r}=0.99$; humidity, $\mathrm{r}=0.99 ; \mathrm{n}=586$ regions).

LMMs were fitted using the lmer function of the lme4 $\mathrm{R}$ package, while tests statistics were calculated using the lmerTest package. Partial $R^{2}$ was computed using the $r 2 \mathrm{glmm} \mathrm{R}$ 
package. Finally, we used a generalized additive model (GAMs, fitted with the R $m g c v$ package) to evaluate the temporal trend of the stringency index at the outbreak date across regions experiencing outbreaks in different periods. For this analysis we used GAMs as we expected a complex temporal pattern and we did not have a priori expectations on the shape of relationship

\section{Results}

COVID-19 growth rates showed high variability at the global scale (Supplement 1, Fig. S2). The observed daily growth rate during the exponential phase was on average $0.22(\mathrm{SD}=0.11, \mathrm{~N}=$ 586 regions), and ranged from $<0.01$ (Argentina, Santiago del Estero and Canada, Prince

Edward Island) to 0.72 (Denmark). The exponential growth phase lasted on average $9.0 \mathrm{~d}(\mathrm{SD}=$ 5.7) and was generally followed by a deceleration of growth, likely as a progressive effect of containment actions and/or increasing awareness by local communities (Supplement 1, Fig. S3) (Maier and Brockmann, 2020). The highest growth rates were observed in temperate regions of the Northern Hemisphere, although relatively fast growth also occurred in some tropical countries, notably Brazil, Indonesia and the Philippines (Supplement 1, Fig. S2). COVID-19 growth rates tended to decrease markedly from March to May (Fig. 1a). At the same time, the stringency of containment measures strongly increased: since the end of March, most outbreaks occurred in regions already under strict containment regimes (Fig. 1b).

Mixed models including environmental and socio-economic variables explained well variation of COVID-19 growth rate across regions (Supplement 1, Fig. S4). Due to collinearity among predictors (Supplement 1, Table S1), we explored different model formulations 
(Supplement 1, Table S2 and Fig. S4). The model including temperature (either 30-days period or $\Delta 14$ days period), its squared term and PM2.5 as environmental variables, and population density, population size and health expenditure as socio-economic predictors showed the best fit during the early outbreaks, and had similar explanatory power to alternative model formulations when we considered later periods (Supplement 1, Fig. S4). We therefore rely on this model as the main basis for subsequent inference.

Temperature was the strongest environmental predictor during early outbreaks, explaining as much as $20 \%$ of the variance in COVID-19 growth rates (Fig. 2). Its effect began to fade when we also included the outbreaks occurring in late March and became negligible from compared to temperature (Fig. 2). Higher PM2.5 levels were associated with fast growth rates when considering early outbreaks only (Fig. 3). Population size and health expenditure were the strongest socio-economic predictors of growth rates (Fig. 2), the highest growth rates being consistently associated with larger population size and greater health expenditure during both early and late outbreaks (Fig. 3). The stringency of containment measures at outbreak onset consistently negatively predicted COVID-19 growth rates (Fig. 3), becoming the predictor with the strongest effect on growth rates from mid-April onwards (Fig. 2). Results obtained using either the 30 -days or the $\Delta 14$ days period were nearly identical (Table S3a-b), even though the model using the 30-days period showed slightly higher fit, and temperature effects during early outbreaks were somewhat stronger when considering the 30 -days period compared to the $\Delta 14$ days period (Fig. 2).

To illustrate the relationships between COVID-19 growth rate and environmental variables, socio-economic variables, or stringency index, we produced partial regression plots 
from models fitted on data up to three time points (March 15, to April 15 and May 15; Fig. 4, Supplement 1, Fig. S5; see Supplement 1, Table S3a for model details). For outbreaks occurring up to March 15, growth rates peaked in regions with mean temperature of ca. $5^{\circ} \mathrm{C}$, decreasing in both warmer and colder climates (Fig. 4a). Furthermore, highly polluted regions experienced a faster disease spread (Fig. 4d). The effects of temperature and air pollution faded completely when including later outbreaks (Fig. 4c-4f). Higher stringency of containment measures consistently reduced growth rates at all three time points (Fig. 4g-i). Considering the effect of airport connections during early outbreaks or considering alternative environmental and socioeconomic variables (absolute humidity, age structure) did not qualitatively alter these conclusions (Supplement 1, Supplementary results and Tables S4-S5).

\section{Discussion}

The role of environmental drivers on COVID-19 spatial patterns and growth rate is controversial (Araújo et al., 2020; Carlson et al., 2020a; Carlson et al., 2020b; National Academies of Sciences Engineering and Medicine, 2020). Some authors suggested that this disease had a reduced impact and spread in warm climates, and in areas with low pollution and experiencing intense UV radiation (Merow and Urban, 2020; Rahman et al., 2020; Runkle et al., 2020; Sajadi et al., 2020;

Our results considering the earliest COVID-19 data only (up to March, 2020) are in line with initial evidence reporting less COVID-19 daily new cases and mortality in warm climates 
(Wu et al., 2020c; Zhang et al., 2020), but exploring a broader time window explained the inconsistency of results across studies. Many previous studies did not explicitly model non-linear when COVID-19 reached an almost global spread (Supplement 1, Fig. S2), and adopted an objective approach to identify the exponential phase of outbreaks (Hall et al., 2014). This 
contact among people increase in warm days). Thus, climate allows predictions of outbreaks of respiratory illnesses (Shaman et al., 2010; Tamerius et al., 2013), acting both as direct and/or indirect effect. The non-linear relationships between COVID-19 growth rate and temperature detected for early outbreaks (Fig. 4a) might be explained by complex interplays between weather-related changes in human social behavior, changes in host susceptibility to the virus, or changes in virus survival and transmission patterns (Fares, 2013). Overall, with no or weak containment measures, seasonal climatic variation may affect the spatial spread and the risk of severe COVID-19 outbreaks (Merow and Urban, 2020; Wu et al., 2020c), as observed for other viral diseases (Shaman et al., 2010; Tamerius et al., 2013; Lowen and Steel, 2014; Baker et al., 2020), for which seasonal oscillations might lead to the worse outcomes during the colder (autumn-winter) months. Nevertheless, containment measures are able to successfully limit COVID-19 outbreaks in all climatic conditions (Maier and Brockmann, 2020), and climate alone is unlikely to accurately predict transmission in future outbreaks.

The effect of air pollution on COVID-19 spread during early outbreaks was weaker than the effect of local climate. In the early stages of the global outbreak, we observed more severe outbreaks in regions with poor air quality, as gauged by their higher PM2.5 levels, in line with studies suggesting that poor air quality may enhance local transmission and may increase COVID-19 related mortality, possibly not independently of local meteorological conditions (Azuma et al., 2020; Bianconi et al., 2020; Rahman et al., 2020; Wu et al., 2020b; Yao et al., 2020; Zhang et al., 2020). Air pollution can influence COVID-19 spread through different pathways. First, several studies have shown a worsening of respiratory symptoms from viral diseases in populations exposed to poor air quality (Domingo and Rovira, 2020). For instance, chronic exposure to PM 2.5 correlates with overexpression of the alveolar ACE-2 receptor, 
leading to more severe COVID-19 infection and increasing the likelihood of poor outcomes

(Frontera et al., 2020; Wu et al., 2020b). Furthermore, the virus can remain viable in aerosols for some hours, thus high pollution levels might increase its transmission (Frontera et al., 2020). Nevertheless, more studies are required to clarify the actual impact of air pollution on COVID19 local spread patterns, as well as to identify the actual biological mechanisms (Wu et al., 2020b).

However, the environmental effects on COVID-19 spread during the 2020 global enforced. Air quality effects became negligible when including outbreaks starting after midMarch, while climate effects lasted a bit longer (until mid-April), but eventually disappeared as well (Fig. 4a-b). From late March onward, most new outbreaks began under severe containment actions (Fig. 1b). A weakening of environmental effects when considering late outbreaks is consistent with the expectation that the enforcement of active containment policies limit the spread potential of the disease and fade associations between climate and disease dynamics (Baker et al., 2020; Maier and Brockmann, 2020).

Analyses of environmental effects on COVID-19 spread have been criticized because SARS-CoV-2 shows a substantial rate of undocumented infections (Li et al., 2020b), and because a high frequency of undocumented cases in some regions (e.g. in Africa) could affect conclusions (Roche et al., 2018; Britton and Tomba, 2019). However, in the early phase of the global outbreak, reported positives largely referred to tested individuals showing COVID-19 symptoms that require hospitalization. Therefore, even though our analyses cannot capture the (unknown) dynamics of asymptomatic infections, they provide information on environmental effects on the spread of symptomatic SARS-CoV-2 cases. Furthermore, our analyses took into 
account health expenditure, which is strongly correlated to the daily testing rate across countries (Supplement 1, Supplementary methods and Fig. S6). The high COVID-19 growth rate in exponential growth, which generally lasted $<10$ days. This limits the possibility that countries with higher health expenditure likely arose because of more efficient early reporting of cases, thus considering health expenditure in the analyses should at least partly account for 'surveillance fatigue' (Romero-Alvarez et al., 2017) affected our results.

Our analyses provide compelling evidence for the effectiveness of policy interventions in limiting disease spread within regions (Maier and Brockmann, 2020). Although our study was not designed to explicitly test the effect of containment actions, it clearly showed that outbreaks starting under strict containment actions were consistently less severe than those starting under no or weak containment actions. This was already evident for the early (up to end of March) outbreaks, and became the main factor explaining variation in COVID-19 growth rates among countries when considering later outbreaks.

Containing COVID-19 outbreaks is undoubtedly one of the biggest societal challenges. The huge variation of COVID-19 growth rates among regions with similar climate and air quality levels highlights that diverse and complex social and demographic factors, as well as stochasticity, may strongly contribute to the severity of local outbreaks, irrespective of environmental effects. The potential socio-economic drivers of COVID-19 outbreak are many (Coelho et al., 2020; Jaffe et al., 2020). Even if we did not manage to model the spatial spread of the disease across regions, we integrated several variables reflecting potential socio-economic drivers. The positive relationship with human population size might be explained by multiple, non-exclusive processes including an easier control of early outbreaks in regions with small 
populations, or the occurrence of more trade and people exchanges in the most populated regions, resulting in multiple infection routes and faster spread (Coelho et al., 2020; Jaffe et al., 2020). However, different socio-economic factors were strongly correlated. For instance, areas with high health expenditure were also inhabited by more people older than 65 years predicts very well international trade of goods and services (Supplement 1, Supplementary emphasize that environmental and containment actions effects were consistent irrespective of the specific combination of socio-economic variables being considering, suggesting that unaccounted socio-economic processes should not bias our findings.

In conclusion, our results suggest that local environmental conditions might have affected COVID-19 spread in the early (but not the late) phase of the global outbreak, and that policy interventions can effectively curb disease spread irrespective of environmental conditions (Islam et al., 2020b; Maier and Brockmann, 2020; Thu et al., 2020). Stringent containment measures thus remain pivotal to mitigate the impacts of SARS-Cov-2 infections (Hellewell et al., 2020; Maier and Brockmann, 2020). Yet, information on environmental drivers of COVID-19 can improve the ability of epidemiological models to forecast the risk and time course of future outbreaks, and to suggest adequate preventive or containment actions (Baker et al., 2020). Studies testing the association between environmental features and COVID-19 spread are a rapidly expanding research area that has been attracting increasing attention (Franch-Pardo et al., 2020; Wu et al., 2020b). The unprecedented nature of the pandemic has promoted a growing number of ecological regression analyses, that have identified multiple complex relationships between COVID-19 spread and transmission patterns and diverse environmental features, 
providing a crucial stimulus to a rapidly evolving area of research (Franch-Pardo et al., 2020; interpretations, as identifying the causal processes linking COVID-19 spread dynamics to environmental features remain challenging, still associations detected in ecological analyses can serve as a key starting point for future investigations during the future evolution of the pandemics (Baker et al., 2020; Wu et al., 2020b).

\section{References}

Al Huraimel, K., Alhosani, M., Kunhabdulla, S., Stietiya, M.H., 2020. SARS-CoV-2 in the environment: Modes of transmission, early detection and potential role of pollutions. Sci. Total Environ. 744, 140946. useful for SARS-CoV-2. Nature Ecol. Evol. on the epidemic growth during COVID-19 outbreak in Japan. Environmental Research 190, 110042.

Baker, R.E., Yang, W., Vecchi, G.A., Metcalf, C.J.E., Grenfell, B.T., 2020. Susceptible supply limits the role of climate in the early SARS-CoV-2 pandemic. Science 369, 315-319. 
Bianconi, V., Bronzo, P., Banach, M., Sahebkar, A., Mannarino, M.R., Pirro, M., 2020.

Particulate matter pollution and the COVID-19 outbreak: results from Italian regions and provinces. Arch. Med. Sci. 16, 985-992.

Bonacich, P., 1987. Power and Centrality: A Family of Measures. American Journal of Sociology 92, 1170-1182.

Britton, T., Tomba, G.S., 2019. Estimation in emerging epidemics: biases and remedies. J. R. 464 Soc. Interface 16, 10. distribution models are inappropriate for COVID-19. Nature Ecol. Evol. 4, 770-771. seasonality must not misguide COVID-19 response. Nat. Commun. 11, 4312. and Applications Center (SEDAC), Palisades, NY.

Microbe 1, e10.

Chipperfield, J.D., Benito, B.M., O'Hara, R., Telford, R.J., Carlson, C.J., 2020. On the inadequacy of species distribution models for modelling the spread of SARS-CoV-2: response to Araújo and Naimi. EcoEvoRxiv, https://doi.org/10.32942/osf.io/mr32946pn. Coelho, M.T.P., Rodrigues, J.F.M., Medina, A.M., Scalco, P., Terribile, L.C., Vilela, B., Diniz, J.A.F., Dobrovolski, R., 2020. Global expansion of COVID-19 pandemic is driven by population size and airport connections. Peerj 8. 
Domingo, J.L., Rovira, J., 2020. Effects of air pollutants on the transmission and severity of respiratory viral infections. Environmental Research 187, 7. real time. Lancet Infect. Dis. 20, 533-534. study evaluating their performance. Ecography 36, 27-46. in the study of COVID-19. A review. Sci. Total Environ. 739, 140033. 81, 255-259.

Fuhrmann, C., 2010. The Effects of Weather and Climate on the Seasonality of Influenza: What We Know and What We Need to Know. Geography Compass 4, 718-730. measures. Proceedings of the National Academy of Sciences 117, 10484. www.bsg.ox.ac.uk/covidtracker, Oxford.

Hall, B.G., Acar, H., Nandipati, A., Barlow, M., 2014. Growth Rates Made Easy. Mol. Biol. Evol. 31, 232-238. 
Hellewell, J., et al., 2020. Feasibility of controlling COVID-19 outbreaks by isolation of cases and contacts. Lancet Glob. Health 8, e488-e496.

Islam, A.R.M.T., Hasanuzzaman, M., Shammi, M., Salam, R., Bodrud-Doza, M., Rahman, modeling approach. Environmental Science and Pollution Research. coronavirus disease 2019: natural experiment in 149 countries. BMJ 370, m2743.

Jaffe, R., Ortiz Vera, M.P., Jaffe, K., 2020. Globalized low-income countries may experience higher COVID-19 mortality rates. medRxiv, 2020.2003.2031.20049122.

Jung, S.M., Akhmetzhanov, A.R., Hayashi, K., Linton, N.M., Yang, Y.C., Yuan, B.Y., Kobayashi, T., Kinoshita, R., Nishiura, H., 2020. Real-Time Estimation of the Risk of Death from Novel Coronavirus (COVID-19) Infection: Inference Using Exported Cases. J. Clin. Med. $9,10$.

Jüni, P., Rothenbühler, M., Bobos, P., Thorpe, K.E., da Costa, B.R., Fisman, D.N., Slutsky, A.S., Gesink, D., 2020. Impact of climate and public health interventions on the COVID-19 pandemic: a prospective cohort study. Canadian Medical Association Journal 192, E566.

Kampf, G., Todt, D., Pfaender, S., Steinmann, E., 2020. Persistence of coronaviruses on inanimate surfaces and their inactivation with biocidal agents. J. Hosp. Infect. 104, 246-251. Kraemer, M.U.G., et al., 2020. The effect of human mobility and control measures on the COVID-19 epidemic in China. Science 368, 493.

Legendre, P., Legendre, L., 2012. Numerical Ecology. Elsevier, Amsterdam. 
Li, Q., et al., 2020a. Early Transmission Dynamics in Wuhan, China, of Novel CoronavirusInfected Pneumonia. N. Engl. J. Med. 382, 1199-1207. undocumented infection facilitates the rapid dissemination of novel coronavirus (SARS-CoV2).

$531 \quad$ Science $368,489$.

Lowen, A.C., Mubareka, S., Steel, J., Palese, P., 2007. Influenza virus transmission is dependent on relative humidity and temperature. PLoS Pathog. 3, 1470-1476.

Seasonality. J. Virol. 88, 7692-7695.

Maier, B.F., Brockmann, D., 2020. Effective containment explains subexponential growth in recent confirmed COVID-19 cases in China. Science 368, 742-746.

Manenti, R., Mori, E., Di Canio, V., Mercurio, S., Picone, M., Caffi, M., Brambilla, M., Ficetola, G.F., Rubolini, D., 2020. The good, the bad and the ugly of COVID-19 lockdown effects on wildlife conservation: Insights from the first European locked down country. Biol. Conserv. 249, 108728.

Merow, C., Urban, M.C., 2020. Seasonality and uncertainty in global COVID-19 growth rates. Proc. Natl. Acad. Sci. USA 117, 27456-27464.

National Academies of Sciences Engineering and Medicine, 2020. Rapid Expert Consultation on SARS-CoV-2 Survival in Relation to Temperature and Humidity and Potential for Seasonality for the COVID-19 Pandemic (April 7, 2020). The National Academies Press. https://doi.org/10.17226/25771., Washington, DC. Pybus, O.G., Tatem, A.J., Lemey, P., 2015. Virus evolution and transmission in an ever more connected world. Proc. R. Soc. B 282, 20142878. 
Rahman, M.S., Azad, M.A.K., Hasanuzzaman, M., Salam, R., Islam, A.R.M.T., Rahman, M.M., Hoque, M.M.M., 2020. How air quality and COVID-19 transmission change under different lockdown scenarios? A case from Dhaka city, Bangladesh. Sci. Total Environ., 143161.

Roche, B., Broutin, H., Simard, F., 2018. Ecology and Evolution of Infectious Diseases: press, Oxford.

Romero-Alvarez, D., Peterson, A.T., Escobar, L.E., 2017. Surveillance fatigue (fatigatio vigilantiae) during epidemics. Revista chilena de infectología 34, 289-290.

Runkle, J.D., Sugg, M.M., Leeper, R.D., Rao, Y., Matthews, J.L., Rennie, J.J., 2020. Short-term effects of specific humidity and temperature on COVID-19 morbidity in select US cities. Sci. Total Environ. 740, 140093.

Sajadi, M.M., Habibzadeh, P., Vintzileos, A., Shokouhi, S., Miralles-Wilhelm, F., Amoroso, A., 2020. Temperature, Humidity, and Latitude Analysis to Estimate Potential Spread and Seasonality of Coronavirus Disease 2019 (COVID-19). JAMA Network Open 3, e2011834e2011834.

Shaman, J., Kohn, M., 2009. Absolute humidity modulates influenza survival, transmission, and seasonality. Proc. Natl. Acad. Sci. USA 106, 3243.

Shaman, J., Pitzer, V.E., Viboud, C., Grenfell, B.T., Lipsitch, M., 2010. Absolute Humidity and the Seasonal Onset of Influenza in the Continental United States. PLoS Biol. 8, 13.

Shammi, M., Bodrud-Doza, M., Towfiqul Islam, A.R.M., Rahman, M.M., 2020. COVID-19

pandemic, socioeconomic crisis and human stress in resource-limited settings: A case from Bangladesh. Heliyon 6, e04063. 
Sobral, M.F.F., Duarte, G.B., da Penha Sobral, A.I.G., Marinho, M.L.M., de Souza Melo, A., 2020. Association between climate variables and global transmission oF SARS-CoV-2. Sci. Total Environ. 729, 138997.

Tamerius, J.D., Shaman, J., Alonso, W.J., Bloom-Feshbach, K., Uejio, C.K., Comrie, A., Viboud, C., 2013. Environmental Predictors of Seasonal Influenza Epidemics across Temperate and Tropical Climates. PLoS Pathog. 9, 12.

Thu, T.P.B., Ngoc, P.N.H., Hai, N.M., Tuan, L.A., 2020. Effect of the social distancing measures on the spread of COVID-19 in 10 highly infected countries. Sci. Total Environ. 742, 140430.

Tucker, P., Gilliland, J., 2007. The effect of season and weather on physical activity: A systematic review. Public Health 121, 909-922.

Viboud, C., Simonsen, L., Chowell, G., 2016. A generalized-growth model to characterize the early ascending phase of infectious disease outbreaks. Epidemics 15, 27-37.

World Health Organization, 2020. Coronavirus disease (COVID-2019) situation reports.

https://www.who.int/emergencies/diseases/novel-coronavirus-2019/situation-reports/ [accessed 1 March 2020].

Wu, J.T., Leung, K., Bushman, M., Kishore, N., Niehus, R., de Salazar, P.M., Cowling, B.J., Lipsitch, M., Leung, G.M., 2020a. Estimating clinical severity of COVID-19 from the transmission dynamics in Wuhan, China. Nature Medicine.

Wu, X., Nethery, R.C., Sabath, M.B., Braun, D., Dominici, F., 2020b. Air pollution and COVID19 mortality in the United States: Strengths and limitations of an ecological regression analysis. Science Advances 6, eabd4049. 
Wu, Y., Jing, W., Liu, J., Ma, Q., Yuan, J., Wang, Y., Du, M., Liu, M., 2020c. Effects of temperature and humidity on the daily new cases and new deaths of COVID-19 in 166 countries. Sci. Total Environ. 729, 139051. particulate matter pollution and case fatality rate of COVID-19 in 49 Chinese cities. Sci. Total Environ. 741, 140396. Yap, T.F., Liu, Z., Shveda, R.A., Preston, D.J., 2020. A predictive model of the temperature600 dependent inactivation of coronaviruses. Applied Physics Letters 117. Zhang, Z., Xue, T., Jin, X., 2020. Effects of meteorological conditions and air pollution on COVID-19 transmission: Evidence from 219 Chinese cities. Sci. Total Environ. 741, 140244. 


\section{$606 \quad$ Acknowledgments}

607 We thank the colleagues of the DISPArati group, especially G. Scarì, for insightful and

608 stimulating discussions. We also thank M. Venegoni, G. Venegoni, V. Longoni, J. G. Cecere and

609 L. Serra for commenting on previous draft of our manuscript, and three anonymous reviewers for

610 insightful suggestions.

611

612

613

Author contributions

614

$615 \quad$ Competing interests

616 None

617

618

Data and materials availability

619 All relevant data have been submitted as supplementary files.

620

621 
Fig. 1. COVID-19 growth rate (a) and stringency of containment measures (b) in regions experiencing COVID-19 outbreaks in different periods. The bold lines represent the fit of a generalized additive model, the shaded area its $95 \%$ confidence band. The figures report data for regions where outbreaks occurred between February 27 and May 31, 2020, as before that date data were sparse (<50 regions experienced outbreaks between January 22 and February 26).

Fig. 2. Temporal variation of the importance of variables in explaining COVID-19 growth rate. We fitted regression models starting from regions experiencing outbreaks up to February 27, until we included all regions experiencing outbreaks up to May 31, 2020 ( $\mathrm{n}=586$ regions). The partial $R^{2}$ statistic (variance explained by each fixed effect) was taken as a measure of the relative importance of variables. a) temperature calculated using the 30-days period; b) temperature calculated using the $\Delta 14$ days period (see Supplement 1 , Fig. S1 for details).

Fig. 3. Temporal variation of the relationships between independent variables and COVID-19 growth rate (standardized coefficients). We fitted regression models starting from regions experiencing outbreaks up to February 27, until we included all regions experiencing outbreaks up to May 31, 2020 ( $\mathrm{n}=586$ regions). The plot includes temperature calculated using the 30days period; the pattern was identical if a $\Delta 14$ days period was used (see Supplement 1, Fig. S1 for details). Shaded areas represent $95 \%$ confidence bands. When confidence bands do not cross the horizontal broken line ( 0 threshold), the effect of a given variable is statistically significant $(P<0.05)$.

Fig. 4. Variation of COVID-19 growth rate in relation to local mean temperature (30-days period), air pollution (PM 2.5) and stringency of containment measures. Partial regression plots from mixed models of COVID-19 mean daily growth rates fitted for local outbreaks occurring up to March 15 ( $\mathrm{n}=195$ regions), April 15 ( $\mathrm{n}=529$ regions) and May 15 ( $\mathrm{n}=577$ regions) are shown. The shaded areas are $95 \%$ confidence bands. 


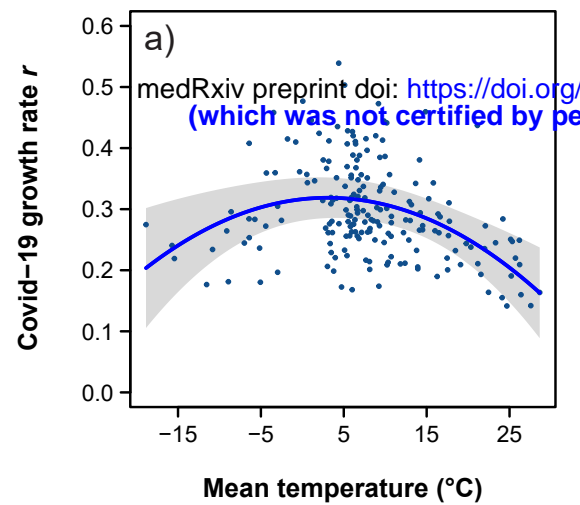

$$
0.6-\text { b) }
$$

\section{b) $\quad 0.6-\frac{}{c)}$}
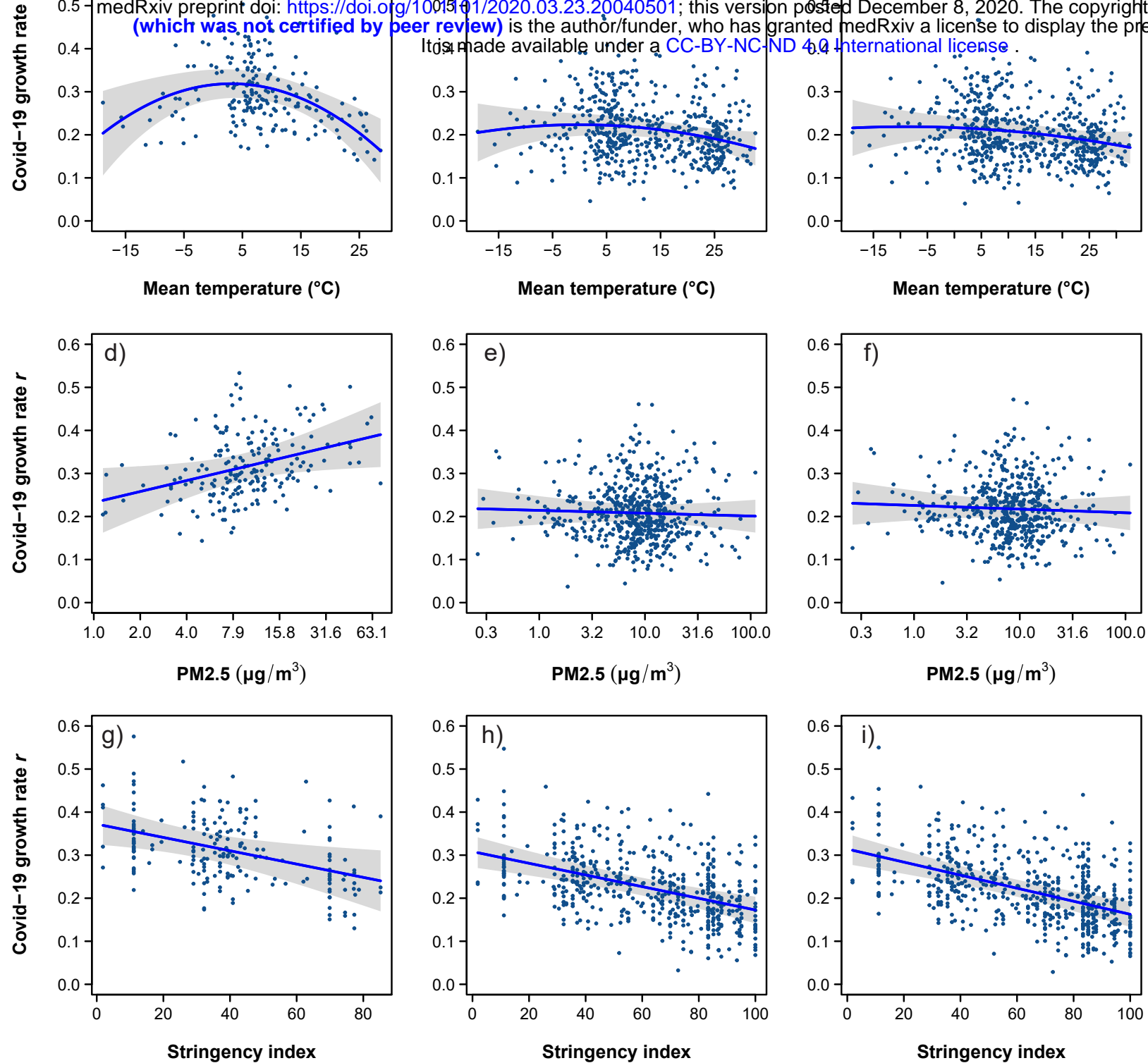
medRxiv preprintgoi htt s: //doi.org/10.1101/2020.03.23.20040501; this version posted December 8, 2020. The copyright holder for this pr.pprint (which was $k 6$ teer ffied by peer review) is the author/funder, who has granted medRxiv a license to display the preprint in perpetuity. a) It is madeavailable under a CC-BY-NC-ND 4.0 International license.
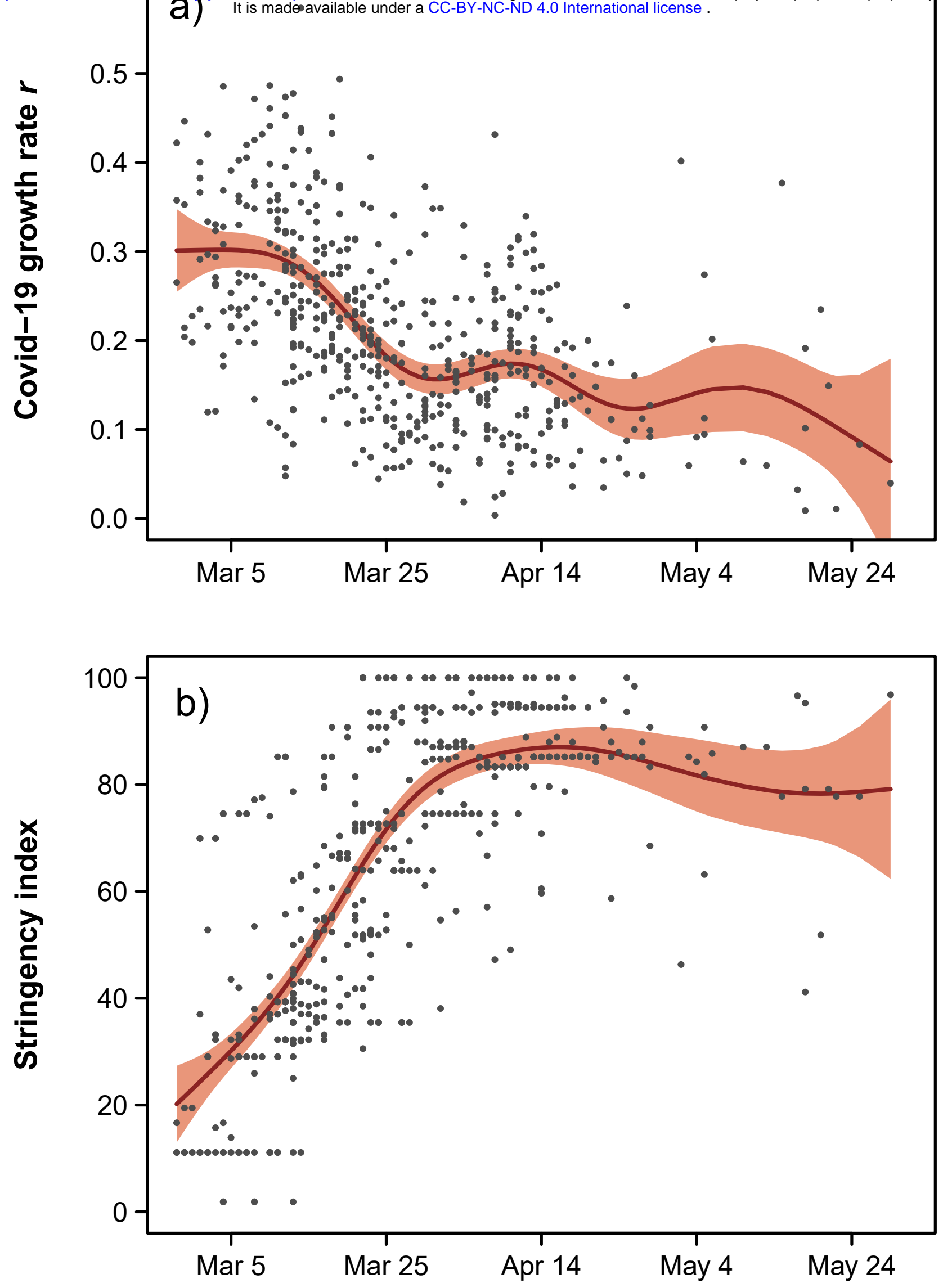


\section{$0.25-$ a) 30-days period temperature}

medRxiv preprint doi: https//doi.org/10.1101/2020.03.23.20040501; this version posted December 8, 2020. The copyright holder for this preptint (which was not certif ed by peer review) is the author/funder, who has granted medRxiv a license to display the preprint in perpetuity.
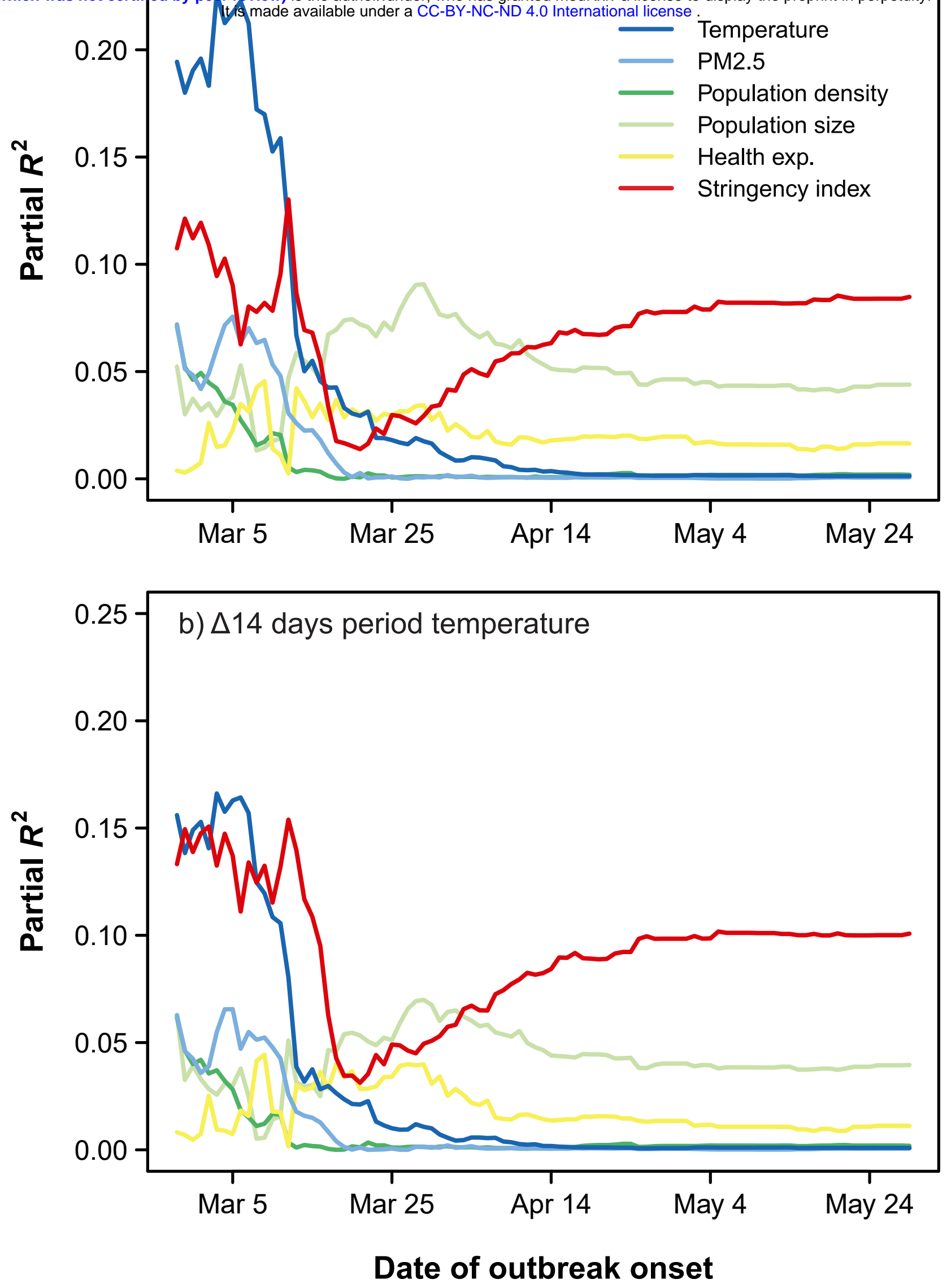

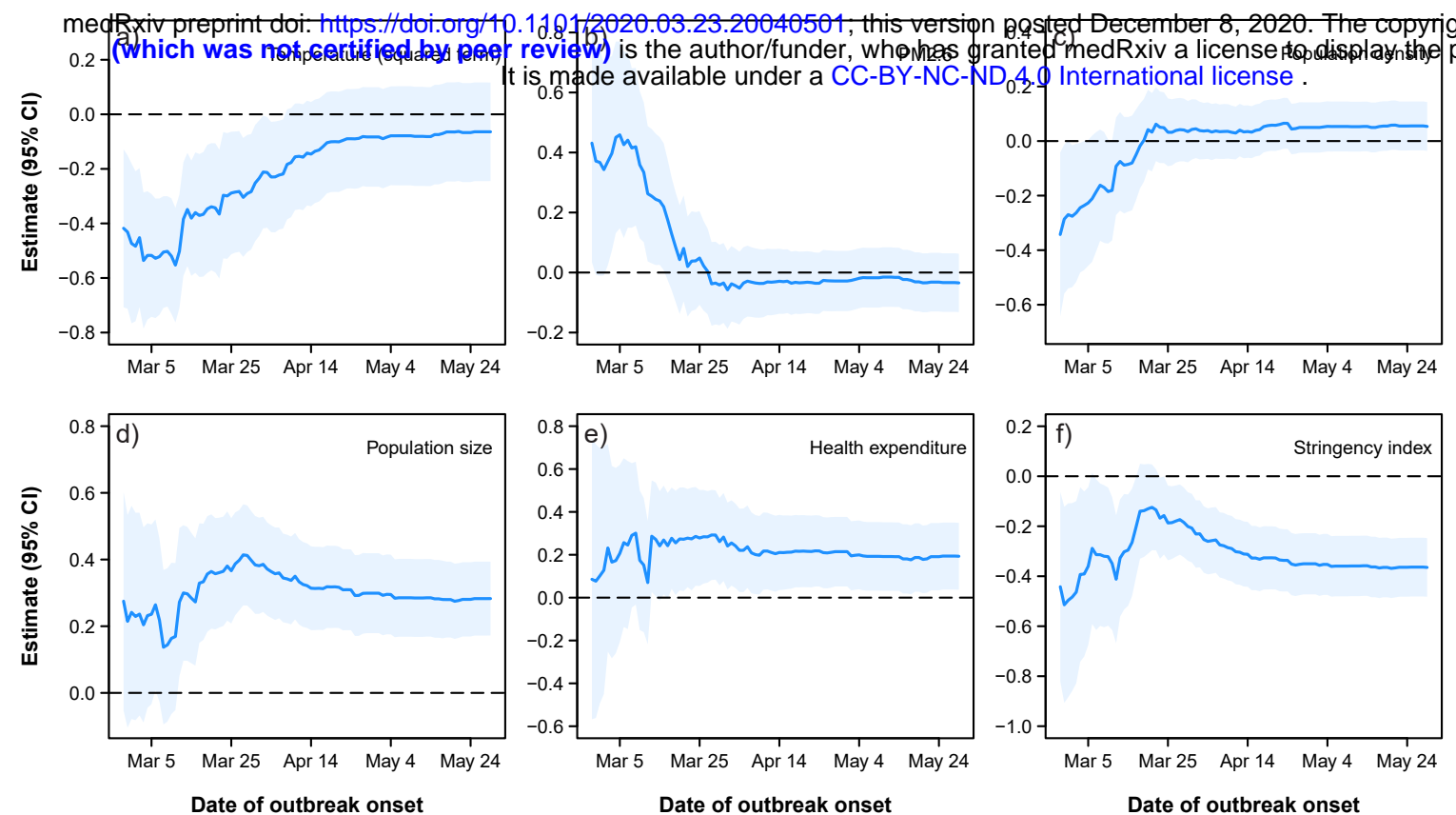

Date of outbreak onset

Date of outbreak onset

Date of outbreak onset 